\title{
The Remarkable Eclipsing Binary TW Draconis
}

\author{
M. Zejda ${ }^{1} \dagger$, Z. Mikulášek ${ }^{1}$, M. Wolf ${ }^{2}$, and P. Svoboda ${ }^{3}$ \\ ${ }^{1}$ Institute of Theoretical Physics and Astrophysics, Faculty of Science, Masaryk University, \\ Kotlářská 2, CZ-611 37 Brno, Czech Republic \\ ${ }^{2}$ Astronomical Institute of the Charles University, Faculty of Mathematics and Physics, \\ V Holešovičkách 2, CZ-180 00 Praha 8, Czech Republic \\ ${ }^{3}$ Výpustky 5, CZ-614 00 Brno, Czech Republic
}

\begin{abstract}
We analyzed a new photometry of this well-known Algol-like eclipsing binary together with old photoelectric measurements with the aim of better understanding of its orbital period changes and short-time light variations modulating the mean light curve. The analysis has been done by the new method based on the combination of the principal component analysis and robust regression. New spectroscopic observations and radial-velocity curve are also presented.
\end{abstract}

Keywords. binaries: eclipsing, oscilations, stars: individual (TW Dra)

\section{Introduction}

TW Draconis $\left(\alpha=15^{\mathrm{h}} 33^{\mathrm{m}} 51^{\mathrm{s}} .1, \delta=63^{\circ} 54^{\prime} 26^{\prime \prime}(2000.0)\right)$ is a well-known and often observed Algol-type eclipsing binary. The variable star is an A-component of visual binary ADS 9706. The light variations of TW Dra are caused predominantly by eclipses of the hot main sequence star A8V by the cooler and fainter giant component K0III. The primary minimum 8.0 - $10.5 \mathrm{mag}$ (spanning about 11.5 hours) and shallow secondary minimum 8.0-8.1 mag are repeating with the period of 2.8068 days. The $O-C$ diagram illustrates large changes of the orbital period in the last 150 years as well as shorter oscillations of it after JD 2432000 (see Figure 1). The system has been studied photometrically and spectroscopically. However, the satisfactory solution of this unique system has not been published up to now.

\section{Photometry}

We have observed this star photometrically using different telescopes equipped with CCD cameras or photoelectric photometers (Brno, Hvar, and Suhora Observatories) since 2003. We have obtained more than 45,000 measurements of brightness in $U B V R I$ so far (see Figure 2). We have confirmed the presence of small variations (hundredths of magnitude) in the light curve on the time scale of tens of minutes. However, their incidence during the total minima rules out any $\delta$ Scuti pulsations of the primary star.

\section{Spectroscopy}

Spectroscopic observations were secured with the 2-m telescope at Ondřejov Observatory. We have obtained 34 high dispersion spectrograms in $\mathrm{H} \alpha$ region (6230-6770 $\mathrm{A}$ ). The secondary component is detectable on some well exposed spectrograms. The following

$\dagger$ E-mail address: zejda@physics.muni.cz 


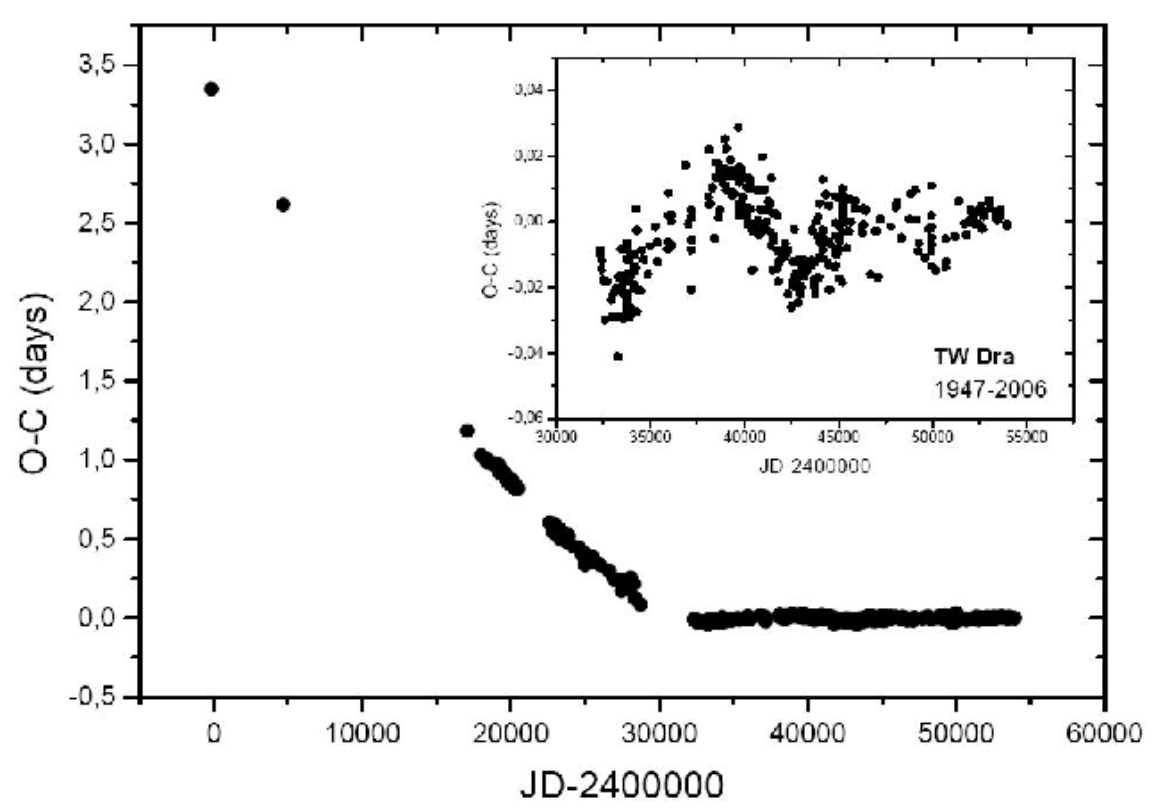

Figure 1. $O-C$ diagram of TW Dra (1858-2006) The dramatic change of the period appeared during World War II. However, the very last change of the period occurred approximately at the end of 2003 (see detailed figure inside).
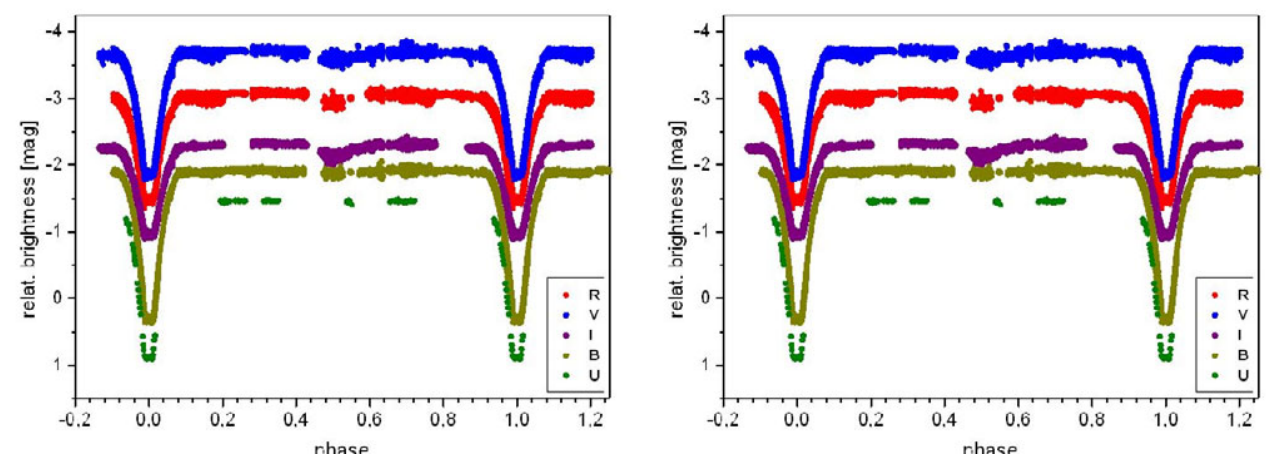

Figure 2. a) Complete light curve of TW Dra. The secondary minimum is not detectable in the blue. b) Small variations on the light curve. Upper panel shows CCD observation made at Brno Observatory (0.4-m+ST7, filter $B)$, the lower panel contains measurements obtained at private observatory of PS (0.07-m+ST7). The amplitude of small oscillations varies between $0.01-0.10$ mag.

spectral lines were detected and measured in Spefo (Škoda 1996): H $\alpha$,Si II, Fe I, Fe II, Ca I. 
TW Dra (2m telescope Ondřejov)
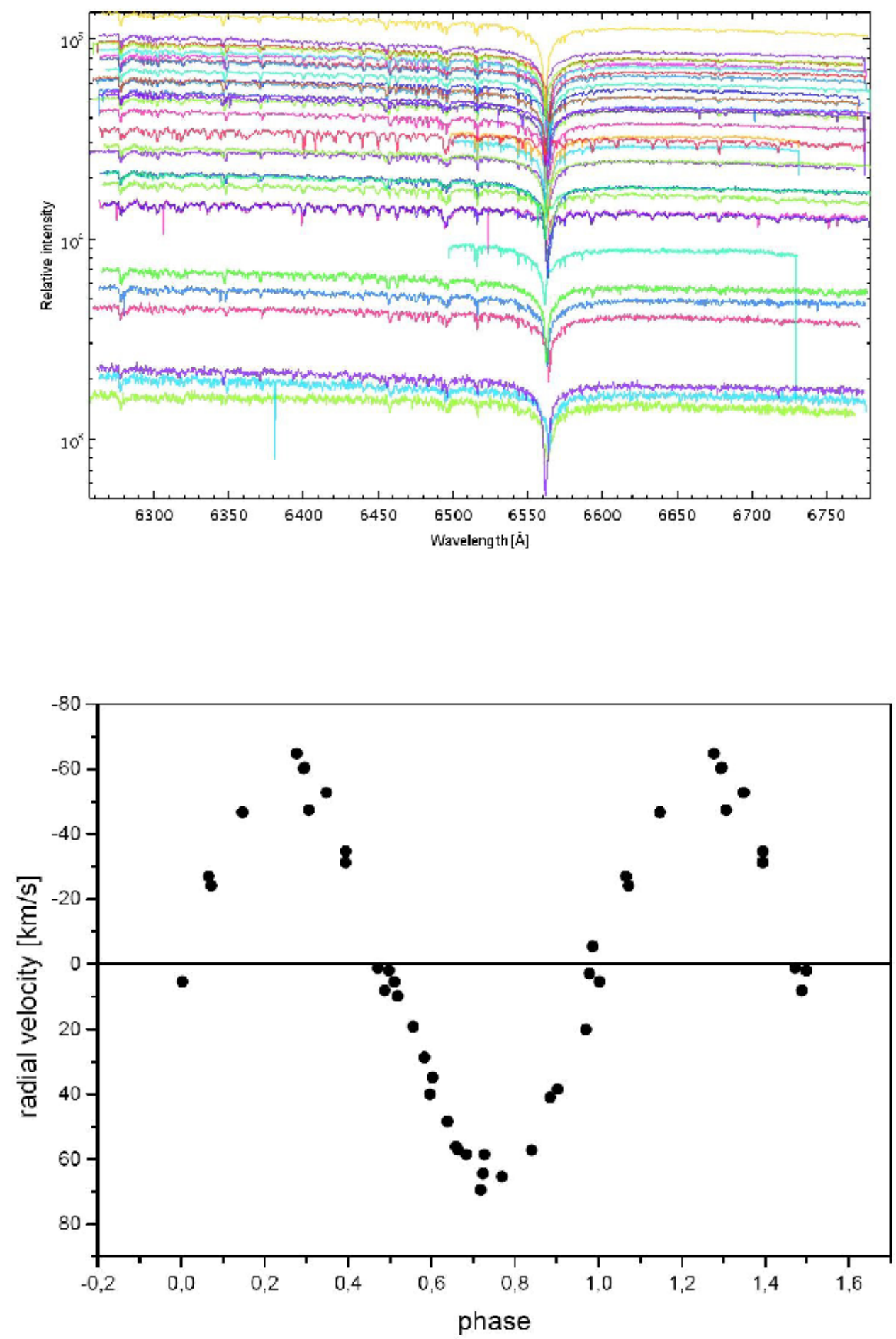

Figure 3. a) Collection of available spectrograms (in $\mathrm{H} \alpha$ region) of TW Dra plotted in Splat-VO (Draper 2001). b) Radial-velocity curve of the primary component with an amplitude of about $70 \mathrm{~km} / \mathrm{s}$. 


\section{Conclusions}

The time-span of period changes and their amplitude seems to be smaller in the last decades. The origin of small oscillations on the light curve is unknown so far. Our detailed light curves and radial velocity curve analyses using Phoebe (Prša 2005) will be published elsewhere.

\section{Acknowledgements}

This investigation was supported by the Grant Agency of the Czech Republic, grants No. 205/04/2063 and No. 205/06/0217.

\section{References}

Draper, P.W. 2001-3, Central Laboratory of the Research Councils, http://www. starlink.ac.uk/splat

Kim, S.-L., Lee, J.W., Kwon, S.-G., Youn, J.-H., Mkrtichian, D.E., \& Kim, C. 2003, A\&A 405, 231

Kusakin, A.V., Mkrtichian, D.E., \& Gamarova, A. Yu. 2001, IBVS 5106

Prša, A. \& Zwitter, T. 2005, ApJ 628, 426

Škoda, P. 1996, Astronomical Data Analysis Software and Systems V, ASP Conf. Series, Vol. 101, G.H. Jacoby \& J. Barnes, eds., p. 187

Zejda, M. 2004, IBVS, 5583 